\title{
Smartphone-based indoor pedestrian tracking using geo-magnetic observations
}

\author{
Sungnam Lee, Yohan Chon and Hojung Cha* \\ Department of Computer Science, Yonsei University, Seoul, Korea
}

\begin{abstract}
With the widespread use of smartphones, the use of location-based services (LBS) with smartphones has become an active research issue. The accurate measurement of user location is necessary to provide LBS. While outdoor locations are easily obtained with GPS, indoor location information is difficult to acquire. Previous work on indoor location tracking systems often relied on infrastructures that are influenced by environmental changes and temporal differences. Several studies have proposed infrastructure-less systems that are independent of the surroundings, but these works generally required nontrivial computation time or energy costs. In this paper, we propose an infrastructure-less pedestrian tracking system in indoor environments. The system uses accelerometers and magnetic sensors in smartphones without pre-installed infrastructure. We reduced the cumulative error of location tracking by geo-magnetic observations at corners and spots with magnetic fluctuations. In addition, we developed a robust estimation model that is tolerant to false positives, as well as a mobility model that reflects the characteristics of multiple sensors. Extensive evaluation in a real environment indicates that our system is accurate and cost-effective.
\end{abstract}

Keywords: Indoor localization system, unconstrained device placements, infrastructure-free

\section{Introduction}

Smartphone-based location tracking has become an active research issue, due to the growing population of smartphone users. The global positioning system (GPS) is commonly used for outdoor location tracking, but the system is hardly applicable to indoor environments. Indoor localization typically relies on infrastructures, such as wireless access points (APs) [1-4], Bluetooth beacons [5], or specifically designed hardware [6,7]. Some approaches have used inertial sensing units to track pedestrian movements [8-11]. In order to reduce drift error when using inertial sensing units, several techniques have been developed to make use of radio beacons [12-14] or environmental features [8,11,14].

Infrastructure-based approaches are easily influenced by changes in infrastructure, and they require offline training. In addition, the cost of infrastructure and the overhead to handle the additional sensors are not trivial. Approaches that use only inertial sensing units have drift error, which is a critical problem, as drift error accumulates as time goes by. The simultaneous localization and mapping (SLAM) approach, with an inertial sensing unit, requires a significant amount of computation; therefore, the scheme is generally inadequate for smartphone applications.

The main goal of our work is to track a user's location in indoor environments. To improve practicality, we do not assume any pre-installed infrastructure (i.e., radio beacons), additional sensor unit, indoor

\footnotetext{
${ }^{*}$ Corresponding author: Hojung Cha, Department of Computer Science, Yonsei University, Seoul, Korea. E-mail: hjcha@ yonsei.ac.kr.
} 
floor map, or offline training. In other words, the system needs to be an unsupervised localization system without user intervention. Here, the technical challenges are (1) to revise the cumulative error without radio beacons or map information and (2) to optimize performance with minimum overhead in terms of computation time and energy consumption.

We propose an infrastructure-less indoor localization system using inertial sensors embedded in a smartphone. The system uses Bayesian filtering with a perpendicular mobility model and geo-magnetic observation. The key insights are that indoor layouts typically include corridors arranged at right angles, and spots with unusual magnetic signals can be used as landmarks. The system tracks a user's location using the dead reckoning (DR) method. The system automatically generates landmarks at corners or spots with significant magnetic fluctuation in order to reduce the accumulated DR error. We designed (1) a perpendicular mobility model, assuming that an indoor environment consists of corridors and corners arranged at right angles and (2) an estimation model using significant changes in magnetic value as observations. The proposed system provides pedestrian tracking regardless of the placement of the smartphone and revises positioning errors without any pre-installed infrastructures.

\section{Related work}

We categorized indoor localization system into three groups: infrastructure-less, infrastructure-aided, and hybrid approaches.

Most of the infrastructure-less systems we reviewed used a DR method, which uses an accelerometer and digital compass. The scheme tracks a relative location from a previous one by determining step length and movement direction; the major concern is to revise the cumulative DR errors. To reduce drift errors, each system used different observations. FootSLAM [8] applied the SLAM approach with foot-mounted IMUs. The scheme used environmental context (e.g., doors, rooms) as observations and determined the movement pattern on a hexagonal grid. Ohtaki et al. [9] used body-mounted inertial sensors and a floor map. The system proposed a probabilistic map matching method with a particle filter. However, the computational overhead is a serious limitation of both systems, and the use of bodymounted sensors is impractical.

Works in $[10,11]$ designed tracking systems that use inertial sensors embedded in the smartphone, not additional sensor units. CompAcc [10] used electronic maps to match the sequence of movement direction with path direction on roads. CompAcc tried to eliminate the dependency on infrastructure, but map data was still required. We had previously designed a DR system [11] that used corridor-corner information to revise the cumulative error. When the pedestrian passed through the same corner more than once, the system revised the paths based on corner identification, using heading and distance information. However, the system was unable to handle outliers caused by erroneous sensor readings. The system only compared the corners using heading and distance parameters; therefore, it was unable to handle false positive errors. Compared to these works, we have designed an estimation model that uses magnetic signals as observations.

Recent works have used geo-magnetic fingerprints for indoor localization. The advantage of geomagnetic data is the coverage: it is available everywhere on Earth. Chung et al. [15] used four magnetic sensors with high sensitivity to estimate fingerprints in a high sampling rate along all directions. However, a smartphone typically contains one magnetic field sensor with low sensitivity, which is not sufficiently accurate for the fingerprinting method.

The infrastructure-aided systems commonly used radio beacons for indoor localization. WiFi APs and cell towers are widely used beacons. RADAR [1] used a WiFi fingerprinting method for indoor 
localization. The system collected received signal strength (RSS) measurements as WiFi fingerprints at known locations. RADAR identified the locations of users based on similarities between previously collected and currently observed fingerprints. PlaceLab [2] collected both WiFi and cell signals along roads, and created a radio map using GPS coordination of access points and cell towers. Moura et al. [3] considered human walking speed and floor maps to estimate the presence probability of user's position with WiFi fingerprints. The WiFi-based approaches perform poorly when the infrastructure is sparsely distributed or changes. Extensive studies have examined the use of various types of radio beacons, such as infrared [6], ultrasound [7], and Bluetooth [5]. These approaches are relatively robust and accurate; however, unlike the WiFi and cell-based systems, the cost of sensor distribution outweighs system performance.

The hybrid approaches used both inertial sensors and radio beacons. SparseTrack [12] conducted error correction in a DR system with sparse infrastructure. When the range measurement from one ultrasound beacon is detected, the system revised accumulated error via a sensor fusion scheme. SmartSLAM [13] applied the SLAM approach by using an inertial sensor and WiFi module in a smartphone. The system tracks a user's location using the DR method and uses WiFi fingerprints as observations. UnLoc [14] used indoor environment features as observations, such as an elevator, a corridor-corner, or a spot with magnetic fluctuation. The system collected such observations by collaborative methods. In contrast to these works, we used geo-magnetic signals as observations, without radio beacons or collaborative collection.

\section{Motivation}

In this section, we elaborate the motivation our work by validating the feasibility of our key insights. The basic assumptions of the proposed system are: (1) an indoor environment is comprised of corridors and corners arranged at right angles; and (2) changes in magnetic signals can be used as distinguishable features at specific locations (i.e., corners or spots with magnetic fluctuation).

To validate the first assumption, we surveyed the indoor layout of 15 major public buildings in Seoul, Korea. We collected floor map data of the buildings, which included department store, outlet, office, museum, and university buildings, and we manually examined the layouts. Among 783 corners in 15 buildings, $88 \%$ of the corners were arranged at right angles. Exceptional corners were observed in lobbies and specially built spaces, such as art museums. The results indicate that the assumption is reasonable for most real buildings.

We then explored the characteristics of magnetic signals in indoor environments to validate the second assumption. Our system used a geo-magnetic signal to generate landmarks. The requirement of a robust landmark is that the patterns at identical positions should be consistent and distinguishable with patterns at different positions. We manually collected geo-magnetic data in four buildings using a Nexus-S smartphone, and estimated the similarities between magnetic signals at every location. We used the root mean square (rms) as the similarity function. It is a widely used metric in the literature, and it is defined as

$$
S\left(M_{i}, M_{j}\right)=\sqrt{\left(m_{i}^{x}-m_{j}^{x}\right)^{2}+\left(m_{i}^{y}-m_{j}^{y}\right)^{2}+\left(m_{i}^{z}-m_{j}^{z}\right)^{2}},
$$

where $M$ is the vector of the magnetic signal $\left(m^{x}, m^{y}, m^{z}\right)$.

Figure 1 shows similarities between magnetic signals in two buildings. A user walked through the same paths twice in each building. The results indicate three intuitive findings. First, the magnetic signals are similar within a straight corridor. This implies that the use of a magnetic sensor embedded in 


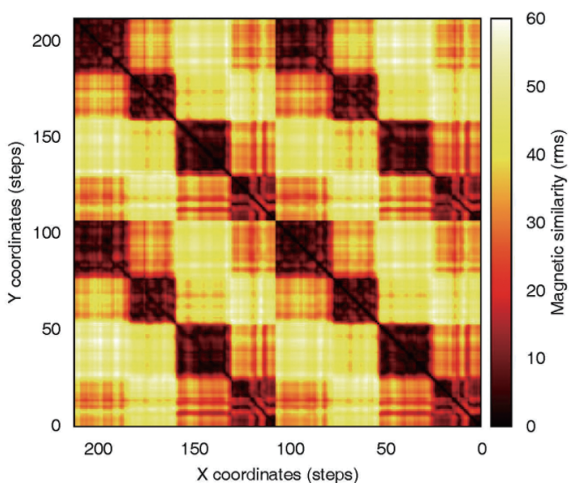

(a)

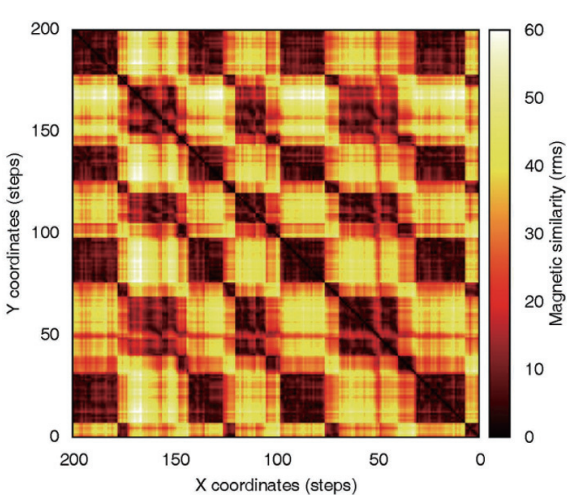

(b)

Fig. 1. Confusion matrix of magnetic signal in two buildings. Low rms value (dark color) indicates that two points show high similarity.

a smartphone is insufficient to distinguish magnetic fingerprints on locations within a straight corridor, although a previous work [15] used four magnetic sensors to estimate magnetic signals along all directions. Second, corners include significant changes in magnetic signals, which can be used as landmarks. Corridors can be segmented as one unit by using corner information. Lastly, spots with significant magnetic fluctuation are observed at specific positions. These spots exhibit a unique signal within a corridor, and the patterns are consistent, as illustrated at around 120 steps along the x-axis in Fig. 1(a) and 50 steps along the $\mathrm{x}$-axis in Fig. 1(b). Based on these findings, our system generated landmarks at corners and spots with significant magnetic fluctuation.

\section{Pedestrian tracking system}

We designed an indoor pedestrian tracking system without any pre-installed infrastructures in indoor environments. The system tracks a user's location using inertial sensors embedded in a smartphone. To revise the drift error, we generated landmarks at spots with significant changes in the magnetic signal. In the following sections, we describe the proposed scheme with the mobility and estimation models.

\subsection{Bayesian filtering}

We define the localization system as the Hidden Markov Model (HMM). As shown in Fig. 2, the hidden state (i.e., the current position) is defined by measurement (position) and the most probable previous observations, which is called a landmark. This condition is defined using a Bayesian filter, which provides a probabilistic estimation of the state, considering the noisy measurements. The Bayesian filter shows the confidence of the system based on a probability distribution, $\mathrm{P}\left(s_{t}\right)$, at time $t$ :

$$
\mathrm{P}\left(s_{t}\right)=P\left(s_{t} \mid u_{1}, \ldots, u_{t}\right)
$$

where $s_{t}$ is a state and $u_{1}, \ldots, u_{t}$ are noisy measurements observed at time $t$.

With the Markov assumption, $\mathrm{P}\left(s_{t}\right)$ is calculated by updating $\mathrm{P}\left(s_{t-1}\right)$, so it is possible to track the states of the system over time. The propagation from $\mathrm{P}\left(s_{t-1}\right)$ to $\mathrm{P}\left(s_{t}\right)$ is defined by the mobility model, which represents the possible transition of the probability distribution from one state to another. The 


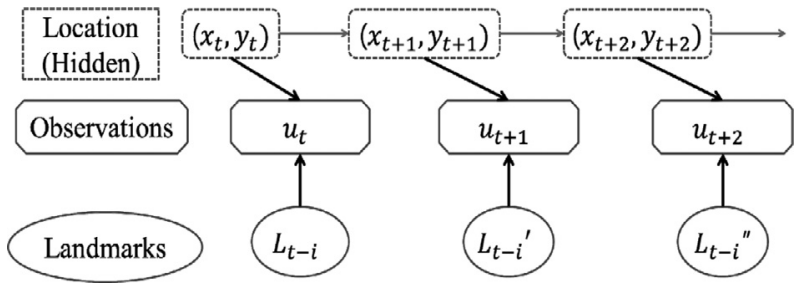

Fig. 2. Hidden Markov model.

reliability of propagation is then corrected with measurements from the system. Each measurement has its own reliability decision model, called an estimation model. The estimation model provides the probability distribution representing the likelihood of the observation at each state. The system then determines the most probable state with given observations.

With the mobility model, the prior probability of the system is now defined as

$$
\mathrm{P}^{\prime}\left(s_{t}\right)=\sum_{s_{t}} p\left(s_{t} \mid s_{t-1}\right) p\left(s_{t-1} \mid u_{1: t-1}\right),
$$

because the probability of the current state can be estimated from the previous states. The updated posterior, therefore, is

$$
\mathrm{P}\left(s_{t}\right)=a_{t} p\left(u_{t} \mid s_{t}\right) \mathrm{P}^{\prime}\left(s_{t}\right),
$$

where $a_{t}$ is the normalization factor and $p\left(u_{t} \mid s_{t}\right)$ is an estimation model with measurement $u_{t}[16,17]$.

The particle filter estimates this distribution with possible weighted samples, called particles:

$$
S_{t}=<s_{t}^{i}, w_{t}^{i}>i=1, \ldots, n,
$$

where $S_{t}$ is the set of particles, $s_{t}^{i}$ the $i^{\text {th }}$ particle, and $w_{t}^{i}$ the corresponding weight. Based on the previous posterior, the particle filter resamples a new posterior. The resampling phase creates a new particle, based on the corresponding weight. A higher weight represents a more probable likelihood function. The new particle propagates to a new state following the definition of the mobility model. Finally, the new particle is weighted with the estimation model. The resampling, transition, and assignment phases are repeated for all particles.

Our system defines the state as

$$
s_{t}=\left(x_{t}, y_{t}, h_{t}, M_{t}, A_{t}\right)
$$

where $x_{t}, y_{t}$ represent the coordination, $h_{t}$ the heading, $M_{t}$ the set of the magnetic field for three axes, and $A_{t}$ the abstract orientation of the device, obtained by accelerometer data. We consider gravity axis and heading axis as an abstract orientation, as suggested in [18].

\subsection{Mobility model}

The transition of state is defined with the mobility model. In our system, the model represents the mobility of a pedestrian, including the fluctuation and error inherent in the sensing data. The user movement consists of two parts - the heading and the distance. The heading is measured with a digital compass and gyroscope, whereas the distance is obtained using an accelerometer. 
Heading measurement. The smartphone sensors have difficulty estimating the heading of a pedestrian. A digital compass provides an azimuth value of the smartphone, but the device is not always aligned with the overall direction of movement.

We calculate relative headings, rather than absolute headings, to handle the placement changes. For indoor environments, determining the path is more important than whether the user is facing north or east. The relative heading, which is relative to the initial heading of the device, makes the pedestrian intuitively interpret where to go from the current position. If the detection of transition from outdoors to indoors is possible, we can obtain an initial heading from recently received GPS data, so that the relative heading matches the global heading.

Because the system uses relative heading for free placement of devices, the sensing data need to be revised when the placement of the device changes. Our previous system detects the change of mount when the gravity axis changes, with correction of the heading value from the previous headings. Therefore, the changes in device placement do not influence the heading value. According to our previous work, the mount detection has a $5.7 \%$ error rate [11].

The mobility model should consider the characteristics of sensing error, such as fluctuation and errors due to steps, surroundings, and presence in the vicinity of other mobile devices. To estimate heading in a robust way, we integrated the gyroscope, which measures angular acceleration when the sensor rotates. However, it is difficult to measure the actual turning angle of a pedestrian, due to noisy measurement and the different axis. The gyroscope data provides the acceleration around the center axis of the device, but the actual turning angle is centered in the user. We used the Kalman filter for combining the gyroscope and digital compass to estimate the heading value. The gyroscope data includes a cumulative error, due to integration, but it is relatively stable. The digital compass, on the other hand, provides instantaneous data without error accumulation, but it has more variations. Based on these characteristics, we applied the Kalman filter to use the gyroscope as a measurement model and the digital compass as an observation model.

Distance measurement. There are several ways to find moving distance. A typical approach is integrating the accelerometer readings twice. However, the integration approach is not adequate for a pedestrianbased system, due to the noisy measurement of smartphone sensors. The step-based method is a popular opportunistic scheme for distance measurement. The number of steps can be detected according to the resulting fluctuations in the accelerometer readings, and with multiplication of stride length, the moving distance of the pedestrian is obtained.

Without user input, the stride length is difficult to measure. We assign some randomness of distance for each particle. Because we use the particle filters, the variation in stride length between different users can be incorporated into each particle. Each particle has a different stride length for each propagation step. The basic stride length is $75 \mathrm{~cm}$, which is an empirically measured average length for normal walking steps [18].

The step count is an important component for distance measurement. Each missed steps accumulates into the distance error; therefore, the accuracy of the step counter directly influences the performance of the whole system. The step counter is implemented with widely known techniques, i.e., peak detection in accelerometer signal. As the pedestrian walks, the accelerometer fluctuates significantly, and the continuous cyclical patterns (i.e., peaks) represent the steps taken. However, instead of simple peak detection, we consider the variation of the accelerometer and the abstract orientation. The abstract orientation represents the gravity axis and the heading axis. The gravity axis is the same as the largest magnitude accelerometer readings. The heading axis represents the axis with the greatest variation of accelerometer readings other than the gravity axis. The peak is detected when the continuous sensor 
data exceeds the variation of the accelerometer. However, the step would still be missed, due to noisy readings. To account for this, the mobility model also considers the omission rate of the step counter, which is about $10 \%$, despite the change of placement. Hence, the system adds one extra step at a $10 \%$ ratio for each particle to account for the missed steps.

\subsection{Estimation model}

The estimation model assigns a weight to each particle to evaluate which particle is more likely to be in the same state as the current measurement. Our system uses the magnetic signal for the estimation model. In corridors, the path is divided into corners and straight lines. The fluctuation and variation at straight lines are handled in the mobility model, so the information at the corners and spots with magnetic fluctuation becomes useful for estimation.

Corner detection. The first type of landmark is generated at corners by observing the heading measurement from the digital compass and gyroscope. Within the predefined window size, the occurrence of radical changes in heading is considered a corner. The accuracy of corner detection varies, depending on the threshold, but we try to remove false negatives and minimize the potential false positives. In this way, the system corrects the path more frequently, but the effect of false positives degrades the performance of the system. Given a heading value $h_{t}$ at time $t$ from mobility model and window size $W$, the system generates a landmark at a location if the difference between the previous heading and the current one exceeds a certain threshold $\delta$, expressed as $\left|h_{t}-h_{t-W}\right|>\delta$.

Spot detection. The second type of landmark is generated at spots with magnetic fluctuation. We estimate these spots by using magnetic and gyroscope signals. The magnetic fluctuation is caused by electrical or metal objects. Thus, the magnetic signal changes significantly at these spots, but the gyroscope indicates stationary headings. Given magnetic signal $m_{t}$, gyroscope value $g_{t}$, and window size $W$, we consider a location as a landmark if

$$
\left|\mu_{t}^{m}\left(m_{t-W+1}, \ldots, m_{t}\right)-u_{t-W}^{m}\left(m_{t-2 W+1}, \ldots, m_{t-W}\right)\right|>\varphi \text { and } \int_{t-2 W}^{t} g \mathrm{~d} g<\varphi,
$$

where $\mu$ is an average value and $\varphi$ is a threshold value. In other words, a location includes a radical change in magnetic signal and stationary gyroscope signal.

Landmark features. We use the magnetic field data and abstract orientation of devices as landmark features. Magnetic field readings indicate the characteristics of locations in indoor environments. We further use the abstract orientation of devices to consider the placement of the smartphone. Because we use one magnetic sensor without offline training, the system cannot estimate the magnetic signal along all directions. Thus, the system considers the abstract orientation of the device as a landmark feature, to match landmarks generated at the same placement. The similarity function between landmarks is defined by the Tanimoto coefficient [19], expressed as

$$
U\left(L_{i}, L_{j}\right)=\left\{\begin{array}{cl}
\frac{M_{i} \cdot M_{j}}{\left\|M_{i}\right\|^{2}+\left\|M_{j}\right\|^{2}-M_{i} \cdot M_{j}} & , \text { if } A_{i}=A_{j}, \\
0 & \text { else }
\end{array}\right.
$$

where $M_{i}$ is a vector of magnetic signal at landmark $L_{i}, A_{i}$ is abstract orientation at landmark $L_{i}$, and the Tanimoto coefficient outputs the similarity between 0 and 1 . The scheme simply generates abstract orientation as the gravity axis and heading axis by using the magnitude of the accelerometer, as suggested in [18]. If the landmarks have a similar heading and are close enough spatially, the system resamples the particles. With the similarity of magnetic field data, we assign weight $w_{t}$ to a particle as $U\left(L_{i}, L_{j}\right)$. The same locations would have greater similarity value; therefore, more weight is assigned to particles with greater similarity. 
Table 1

Experimental environment

\begin{tabular}{lccccc}
\hline Type & Number of buildings & Number of sites & Number of APS & Density of people & Openess of spaces \\
\hline Office (a) & 2 & 6 & 70 & Low & Close \\
Hospital (b) & 2 & 6 & 10 & High & Close \\
Electronics plaza (c) & 3 & 5 & 75 & High & Open \\
Department store (d) & 2 & 6 & 35 & High & Open \\
\hline
\end{tabular}

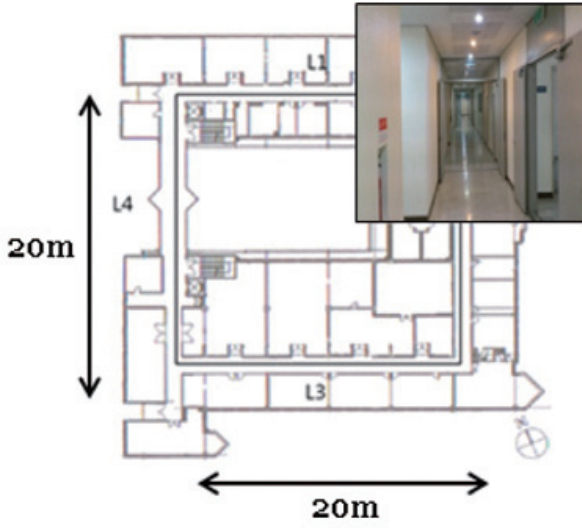

(a)

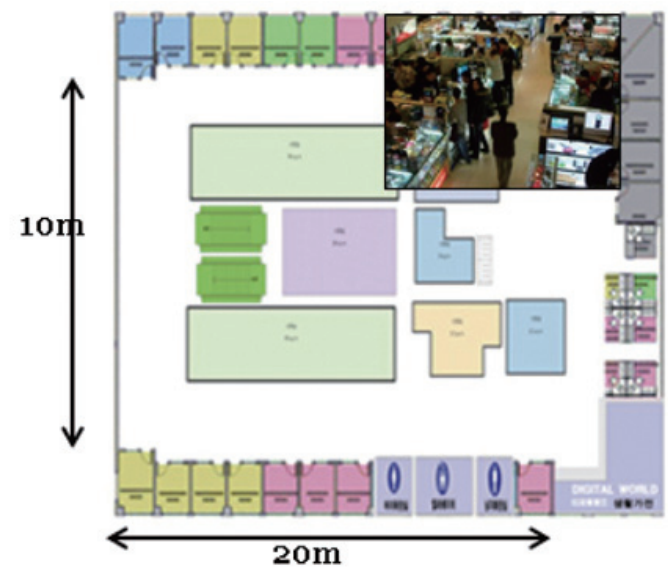

(c)

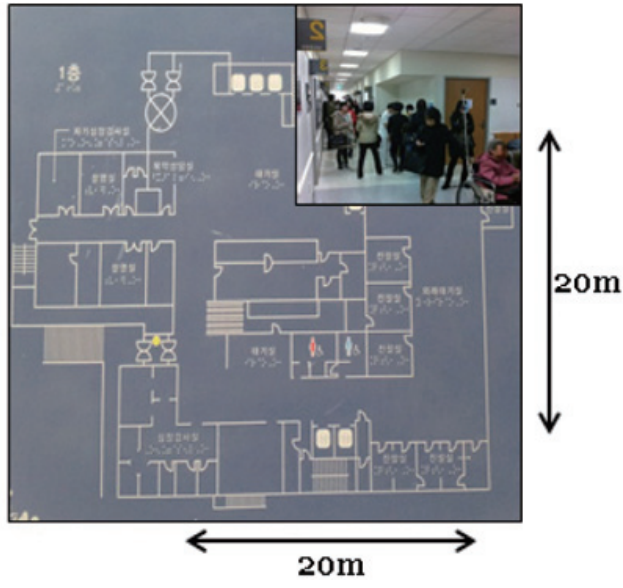

(b)

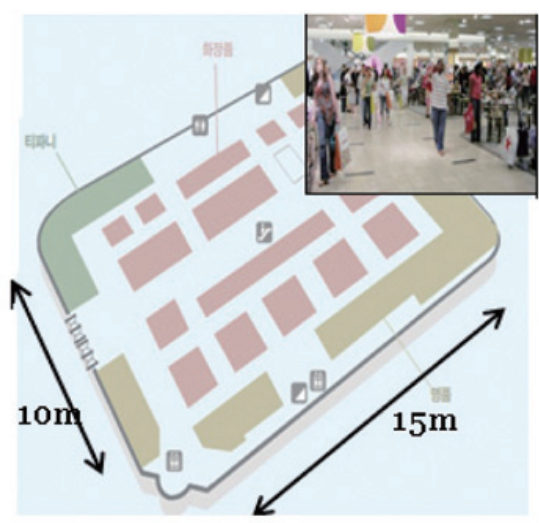

(d)

Fig. 3. Layouts of experimental environments.

\section{Evaluation}

\subsection{Experimental environment}

We implemented the system on the Android platform, using a Nexus S smartphone. We chose 23 sites at four buildings as experimental environments, as described in Table 1. To choose appropriate environ- 


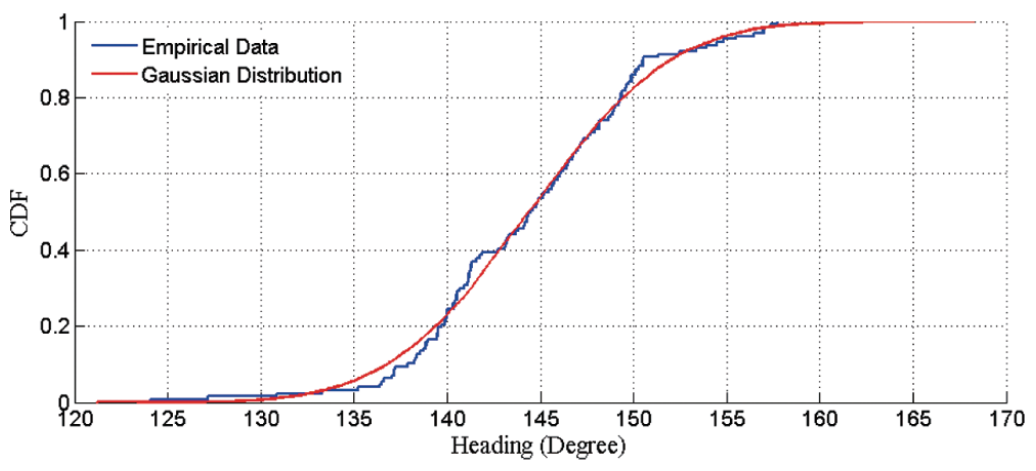

Fig. 4. Distribution of digital compass data.

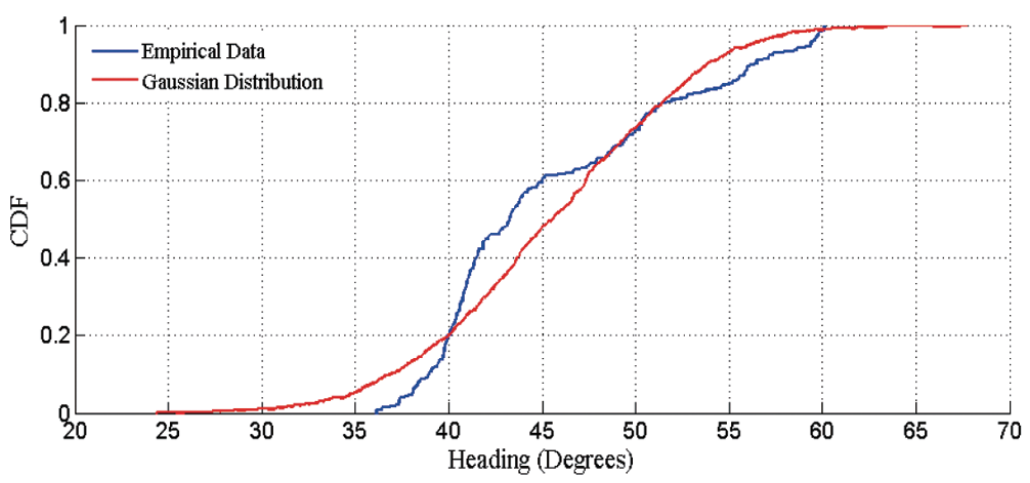

Fig. 5. Distribution of gyroscope data.

ments, we considered three conditions: number of people in spaces, openness of spaces, and number of WiFi APs. The walls around the path are constructed of various materials, including stone, metal, glass, concrete, and plastic. There are elevators in several locations, which could influence the sensor readings. The participants walked the same paths multiple times. The experimental environment is shown in Fig. 3.

\subsection{Micro benchmarks}

We first evaluated the performance of each component. We investigated the sensor errors of the digital compass and gyroscope to handle the noise in the mobility model. We segmented the overall paths into straight lines to explore the sensor errors in a straight line. The result indicates that the error of the digital compass has approximately 20 degrees of standard deviation, and it follows the Gaussian distribution, as shown in Fig. 4. The blue line shows the cumulative distribution function (CDF) of Gaussian distribution, and the red line shows the CDF for collected heading data. To compare the similarity of the two CDFs, we ran the Kolmogorov-Smirnov test (KS test) [20]. The KS test is passed with $\mathrm{P}=0.18$ at $95 \%$ confidential interval. This result shows that it is possible to estimate the distribution of sensor readings with the Gaussian model. The heading measurement characteristic with the gyroscope is shown in Fig. 5 . Similar to a digital compass, the gyroscope measurement also follows the Gaussian distribution. The KS test is passed with $\mathrm{P}=0.23$ at $95 \%$ confidence interval. 


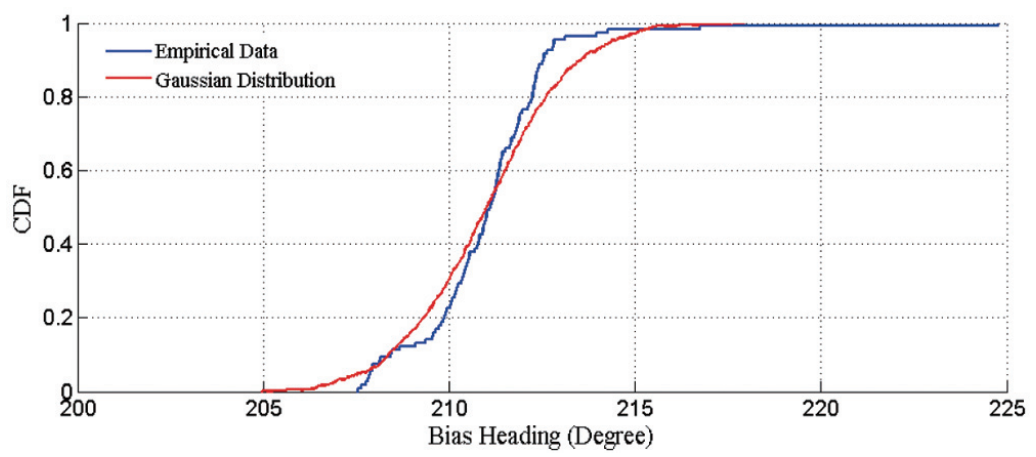

Fig. 6. The distribution of bias error.

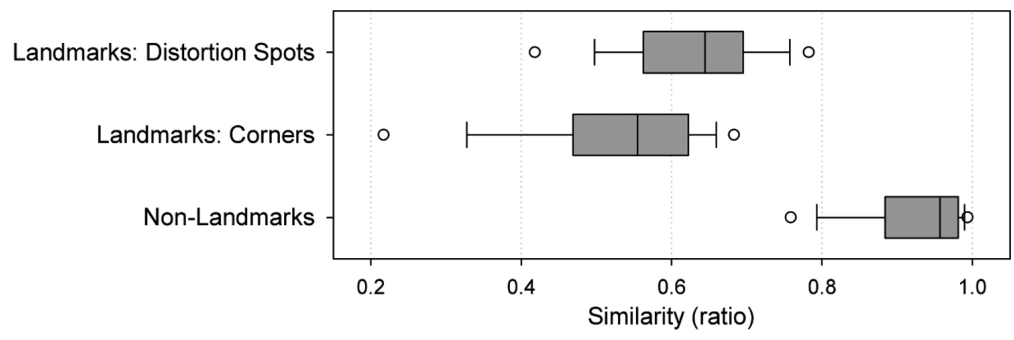

Fig. 7. Landmark(corner/spot) versus non-Landmark.

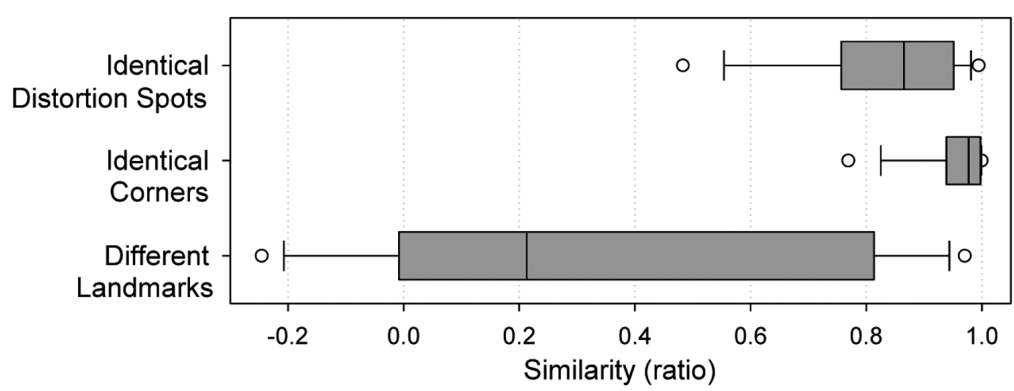

Fig. 8. Similarity of different landmark and same landmark (corner/spot).

The standard deviations of heading measurement error in a straight line are 10.2 degrees for the digital compass and 6.7 degrees for the gyroscope. The standard deviation error after applying the Kalman filter is 4.2 degrees, which is $58.4 \%$ less than the digital compass and $36.9 \%$ less than the gyroscope. The mobility model must also consider the bias of digital compass readings. The measurements after making a turn and after a change in device placement cause a bias error in digital compass readings. As shown in Fig. 6, the bias error is also distributed similar to the Gaussian distribution. The KS test is passed with $\mathrm{P}=0.32$ at $95 \%$ confidence interval. Therefore, for transition and resample, the use of Gaussian distribution based on current heading measurement is reasonable in the estimation model. The proposed system generates landmarks using the change in magnetic signals at corners and spots with magnetic fluctuation. To determine the threshold of magnetic signals, we explored the similarity values between landmark points and non-landmark points. The ground truth of landmark location was manually estimated. Figure 7 presents the distribution of similarities between locations. The result indicates that 


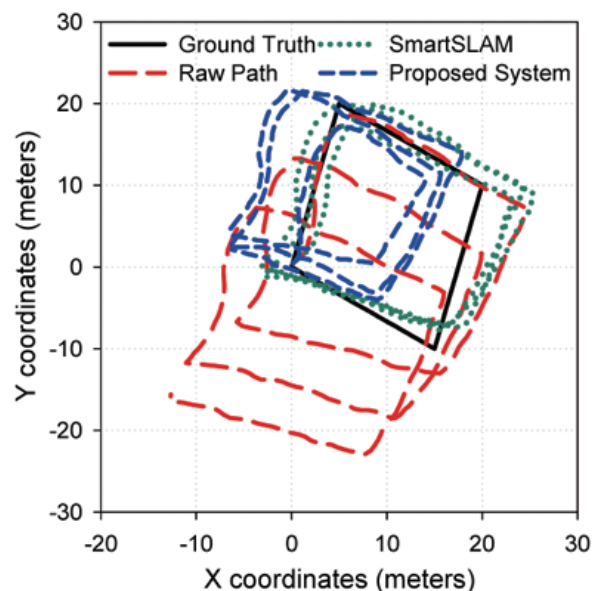

(a)

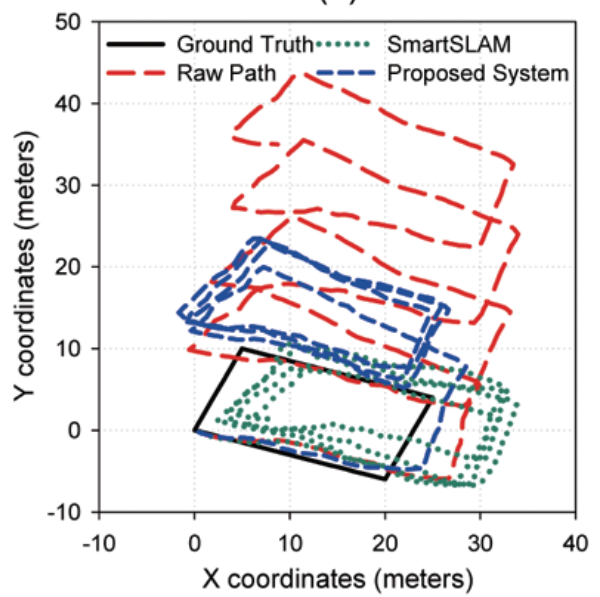

(c)

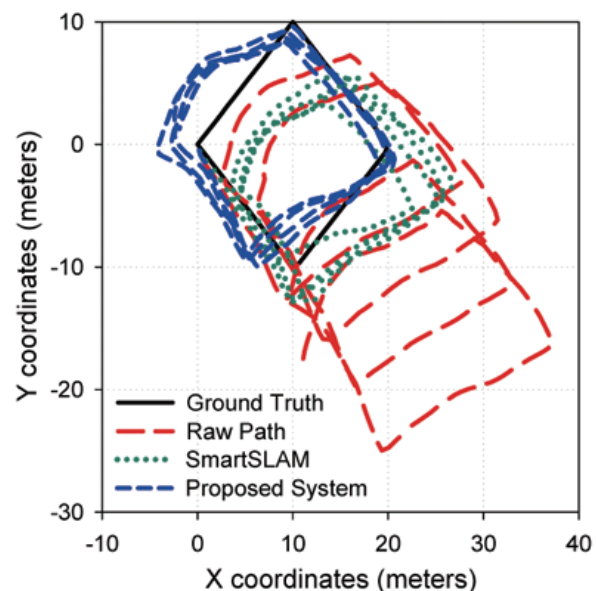

(b)

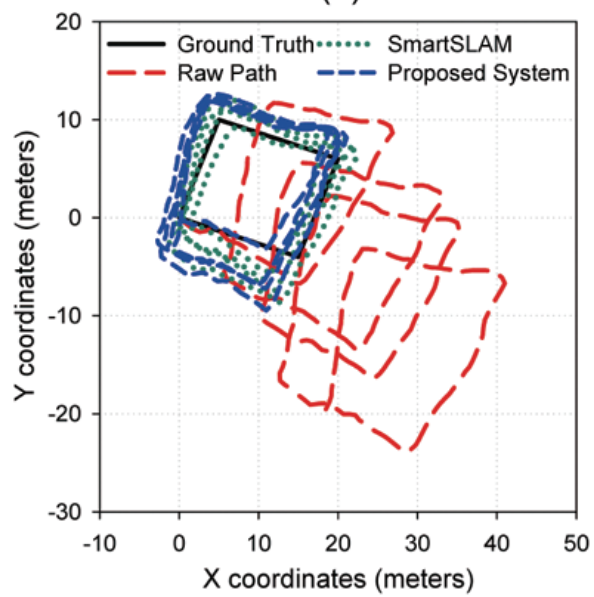

(d)

Fig. 9. Tracking result at four buildings: (a) office, (b) hospital, (c) electronic plaza, (d) department store.

landmark points can be distinguished from non-landmark points. Because false negative (FN) errors are more critical than false positive (FP) ones, we decided empirically on 0.7 as the threshold.

We validated the discrimination power of landmarks. The features of landmarks at the same position should be consistent, and landmarks at different positions should be distinguishable, for robust filtering. The result in Fig. 8 shows that magnetic signals are sufficient to estimate identical landmarks. Intuitively, corners showed clearer features than spots. Spots still includes unique features to be used as a landmark.

\subsection{Macro benchmarks}

We evaluated the overall accuracy and cost of the proposed system. The accuracy metric is the tracking error in meters, and the cost is energy consumption of the smartphone. We compared our system with the following two methods.

Raw Path. This scheme uses a simple DR method, using an accelerometer and a magnetometer without any filtering technique. The method only used the mobility model, without the estimation model. The scheme shows noisy results as baseline. 


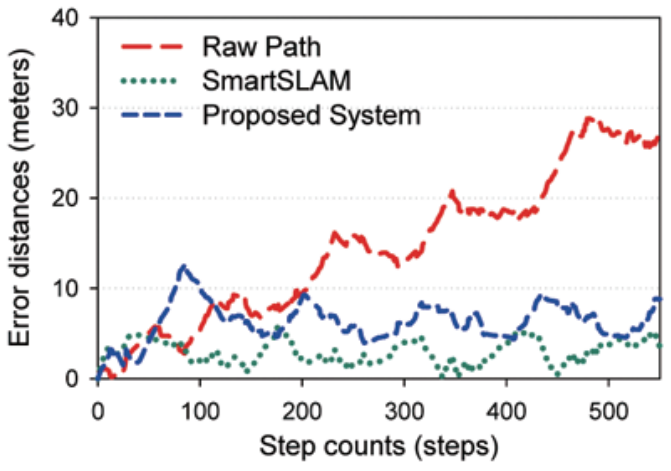

(a)

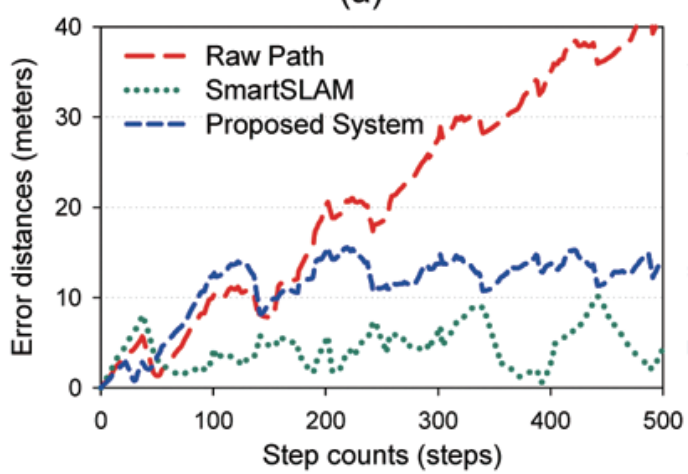

(c)

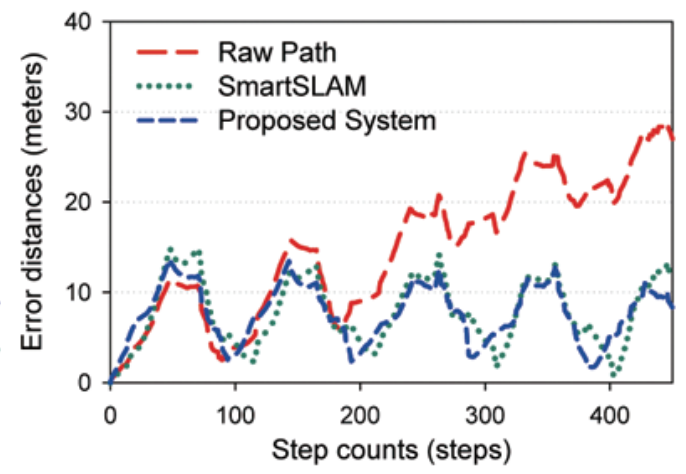

(b)

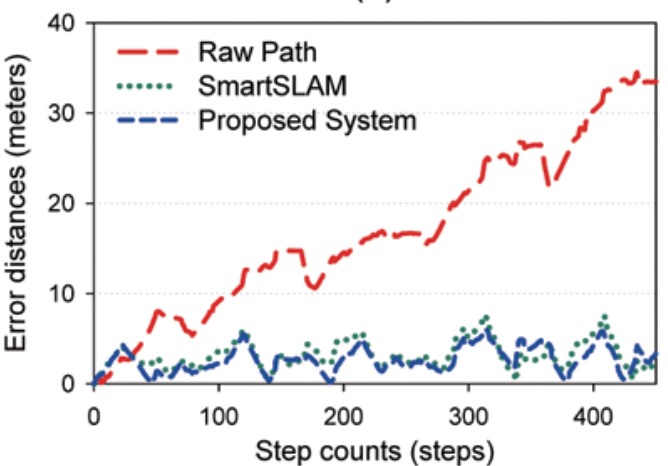

(d)

Fig. 10. Error of related systems.

SmartSLAM [13]. This scheme uses a SLAM technique with a DR method. It uses WiFi fingerprints as observations.

Figure 9 shows the tracking result of the related systems in four buildings. The raw path is significantly biased, due to the accumulated error. SmartSLAM and the proposed system revised the drift error by using WiFi fingerprints and magnetic signals, respectively. The proposed system shows better results at a hospital, where the number of WiFi APs is small, while SmartSLAM derived more accurate results if the number of WiFi APs is sufficient. The accumulated error is shown in Fig. 10. The proposed system revised the accumulated error within a certain bound, while the error of the raw path is continuously increased as time goes by.

Considering the error distance of 26 test cases, SmartSLAM showed smaller errors than the proposed system, as illustrated in Fig. 11(a)-(c). The proposed system showed errors of less than $8.7 \pm 6.1$ meters in $80 \%$ of the cases, while SmartSLAM showed errors of less than $6.7 \pm 5.6$ meters in $80 \%$ of the cases. Figure 11(b) indicates that the proposed system slightly outperforms other systems in the regions with fewer WiFi APs. Despite similar tracking results, the proposed system consumes significantly less energy than SmartSLAM, as shown in Fig. 11(d). To estimate the energy consumption of the related systems, we used the energy profile of HTC Desire provided in [21]. The proposed system consumes only $84.3 \mathrm{~J}$ for 10 minutes tracking, which is $49.8 \%$ less than SmartSLAM and $17.9 \%$ more than the raw path. The reason is that the proposed system uses only inertial sensors, which consume a trivial amount of energy, while SmartSLAM requires continuous use of WiFi sensors, consuming relatively greater amounts of energy. Considering the accuracy and energy costs, the proposed system is an efficient way 


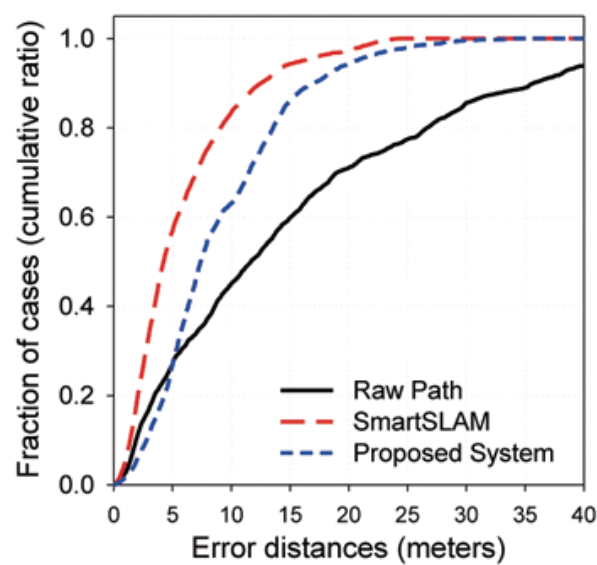

(a)

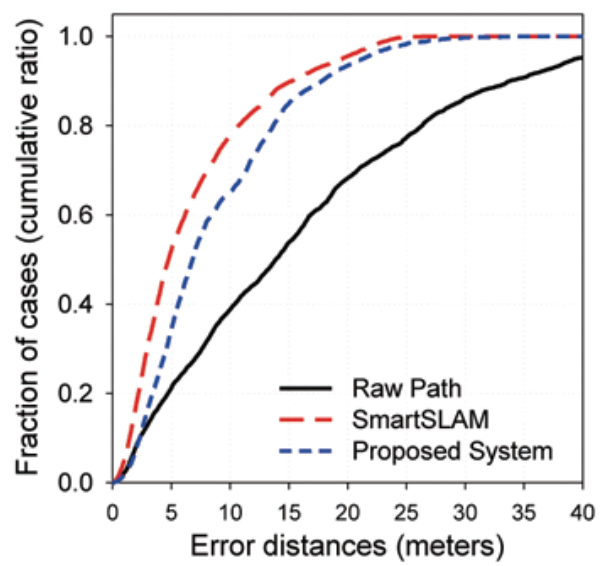

(c)

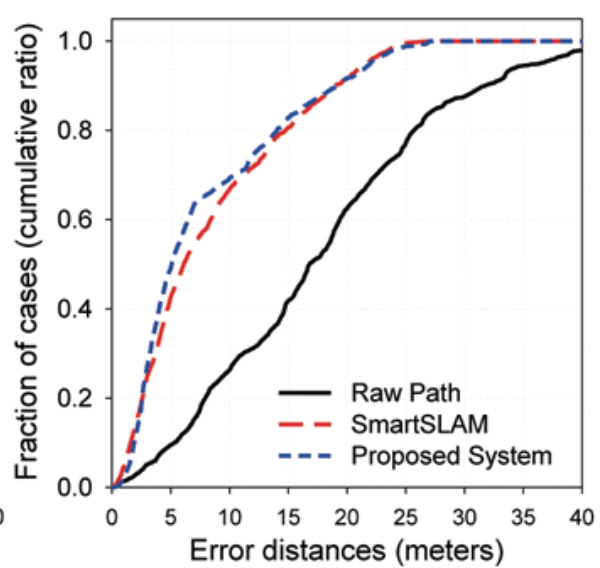

(b)

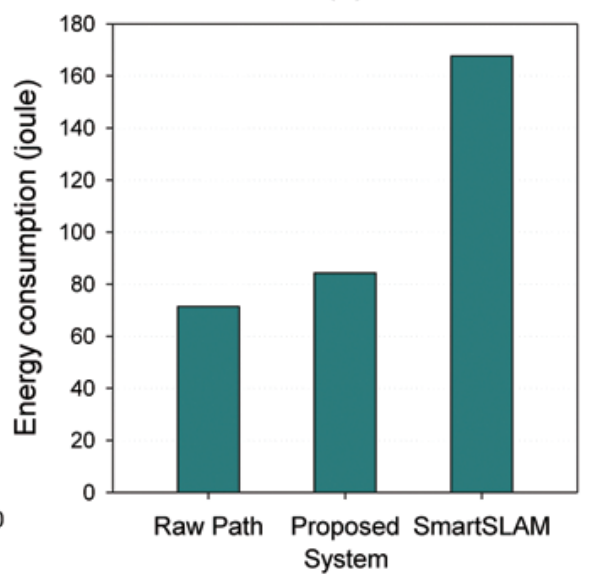

(d)

Fig. 11. Error of related systems.

to track a user's location. In addition, the advantage of the proposed system is that it does not require any pre-installed infrastructures, including WiFi APs.

\section{Conclusion}

In this paper, we proposed an indoor positioning system that does not require additional infrastructure, yet corrects its position with a robust estimation model using magnetic signals. The proposed mobility model reflects the characteristics of multiple sensors that are commonly incorporated into current smartphones. The estimation model using a magnetic field has been verified with various experiments. The overall result is that employing the multi-sensor mobility model and the estimation model with magnetic field data derived accurate tracking results with trivial energy consumption. The contributions of our work are to propose a lightweight, smartphone-based indoor tracking system without infrastructures.

The current corner detection mechanism has limitations. The corner is detected when the pedestrian turns the corner at a right angle; therefore, when users are in a large hall, the system may not work, as pedestrians turn and move around more frequently in large spaces. Thus, a place where unpredictable 
movement occurs continuously may be interpreted as a hall, within some uncertainty boundaries. When the pedestrian leaves the hall and walks along corridors, our system can track the user. We plan to work to refine the distinction between hall and regular movements.

\section{Acknowledgments}

This work was supported by the National Research Foundation of Korea (NRF) grant funded by the Korean government, Ministry of Education, Science and Technology under Grant No. 2012-0005522.

\section{References}

[1] P. Bahl and V.N. Padmanabhan, RADAR: An in-building RF-based user location and tracking system, in: IEEE INFOCOM 2000. Nineteenth Annual Joint Conference of the IEEE Computer and Communications Societies. Proceedings, 2000, Vol. 2, pp. 775-784.

[2] A. LaMarca et al., Place lab: Device positioning using radio beacons in the wild, Pervasive Computing (2005), 116-133.

[3] A.I. Moura, C.H.C. Ribeiro and A.H.R. Costa, WBLS: A signal presence-based Wi-Fi localisation system for mobile devices in smart environments, International Journal of Knowledge-based and Intelligent Engineering Systems 13 (2009), $5-18$.

[4] K. Chintalapudi, A. Padmanabha Iyer and V.N. Padmanabhan, Indoor localization without the pain, in: Proceedings of the sixteenth annual international conference on mobile computing and networking - MobiCom '10, Chicago, IL, 2010, p. 173.

[5] M. Munoz-Organero, P.J. Munoz-Merino and C.D. Kloos, Using bluetooth to implement a pervasive indoor positioning system with minimal requirements at the application level, Mobile Information Systems 8 (2012), 73-82.

[6] R. Want, A. Hopper, V. Falcão and J. Gibbons, The active badge location system, ACM Transactions on Information Systems 10 (1992), 91-102.

[7] N.B. Priyantha, A. Chakraborty and H. Balakrishnan, The Cricket location-support system, in: Proceedings of the 6th annual international conference on mobile computing and networking - MobiCom '00, Boston, MA, 2000, pp. 32-43.

[8] P. Robertson, M. Angermann and B. Krach, Simultaneous localization and mapping for pedestrians using only footmounted inertial sensors, in: Proceedings of the 11th international conference on Ubiquitous computing - Ubicomp '09, Orlando, FL, 2009, p. 93.

[9] Y. Ohtaki, D. Hu, K. Hashimoto and H. Inooka, A method of personal positioning for indoor customer tracking utilizing wearable inertial sensors, International Journal of Applied Electromagnetics and Mechanics 36 (2011), 75-83.

[10] I. Constandache, R.R. Choudhury and I. Rhee, CompAcc: Using mobile phone compasses and accelerometers for localization, in: INFOCOM, San Diego, CA, 2010.

[11] K. Park, H. Shin and H. Cha, Smartphone-based pedestrian tracking in indoor corridor environments, Personal and Ubiquitous Computing, Springer-Verlag, available on-line, 2012, DOI: 10.1007/s00779-011-0499-5.

[12] Y. Jin, M. Motani, W.S. Soh and J. Zhang, SparseTrack: Enhancing indoor pedestrian tracking with sparse infrastructure support, in: 2010 Proceedings IEEE INFOCOM, 2010, pp. 1-9.

[13] H. Shin, Y. Chon and H. Cha, Unsupervised construction of indoor floor plan using smartphone, IEEE Transactions on Systems, Man, and Cybernetics, Part C: Applications and Reviews (SMCC) 42(6) (November 2012), 889-898.

[14] H. Wang, S. Sen, A. Elgohary, M. Farid, M. Youssef and R.R. Choudhury, No need to war-drive: Unsupervised indoor localization, in: Proceedings of the 10th international conference on mobile systems, applications, and services, MobiSys '12, 2012, Lake District, UK, pp. 197-210.

[15] J. Chung, M. Donahoe, C. Schmandt, I.-J. Kim, P. Razavai and M. Wiseman, Indoor location sensing using geomagnetism, in: Proceedings of the 9th international conference on mobile systems, applications, and services MobiSys '11, Bethesda, MD, 2011, p. 141.

[16] D. Fox, KLD-Sampling: Adaptive particle filters, Advances in Neural Information Processing Systems 14 (2001), $713-$ 720.

[17] S. Russell and P. Norvig, Artificial Intelligence: A Modern Approach, 3rd ed. Prentice Hall, 2009.

[18] Y. Chon and H. Cha, LifeMap: A smartphone-based context provider for location-based services, IEEE Pervasive Computing 10 (2011), 58-67.

[19] P. Jaccard, The distribution of the flora in the alpine zone, New Phytologist 11 (1912), 37-50.

[20] G.R. Shorack and J.A Wellner, Empirical Processes with Applications to Statistics, Society for Industrial and Applied Mathematics, 2009 
[21] Y. Chon, E. Talipov, H. Shin and H. Cha, Mobility prediction-based smartphone energy optimization for everyday location monitoring, The 9th ACM Conference on Embedded Networked Sensor Systems (SenSys 2011), Seattle, WA, November 2011.

[22] Y.-C. Cheng, Y. Chawathe, A. LaMarca and J. Krumm, Accuracy characterization for metropolitan-scale Wi-Fi localization, in: Proceedings of the 3rd international conference on mobile systems, applications, and services, MobiSys '05, Seattle, WA, 2005, p. 233.

[23] H. Zang, F. Baccelli and J. Bolot, Bayesian inference for localization in cellular networks, in: 2010 Proceedings IEEE INFOCOM, 2010, pp. 1-9.

[24] C. Figuera, J.L. Rojo-Álvarez, I. Mora-Jiménez, A. Guerrero-Curieses, M. Wilby and J. Ramos-López, Time-space sampling and mobile device calibration for WiFi indoor location systems, IEEE Transactions on Mobile Computing 10 (2011), 913-926.

Sungnam Lee is currently a Ph.D. candidate at the Department of Computer Science, Yonsei University, Korea. He received his B.Sc. degree in computer science from Korea National Defense University in 2009. His research interests include mobile and ubiquitous computing, context-aware systems, and smartphone-based localization system.

Yohan Chon received the B.S. and M.S. degrees, in 2009 and 2011, respectively, from Yonsei University, Korea, where he is currently working toward Ph.D. degree at the Department of Computer Science. His research interests include mobile sensing systems, human mobility prediction, and context-aware pervasive computing.

Hojung Cha is currently a professor in computer science at Yonsei University, Korea. His research interests include wireless and mobile systems, embedded operating power-aware mobile computing systems. He received his B.S. and M.S. in computer engineering from Seoul National University, Korea, in 1985 and 1987, respectively. He received his Ph.D. in computer science from the University of Manchester, England, in 1991. 

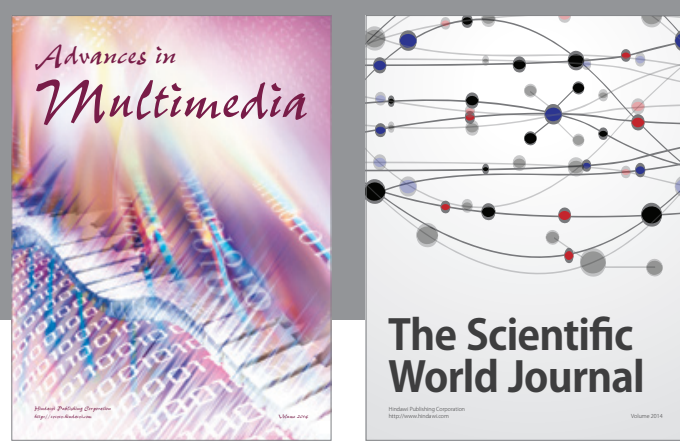

The Scientific World Journal
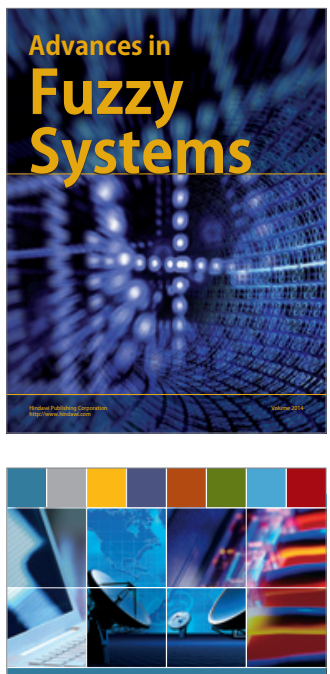

Computer Networks and Communications
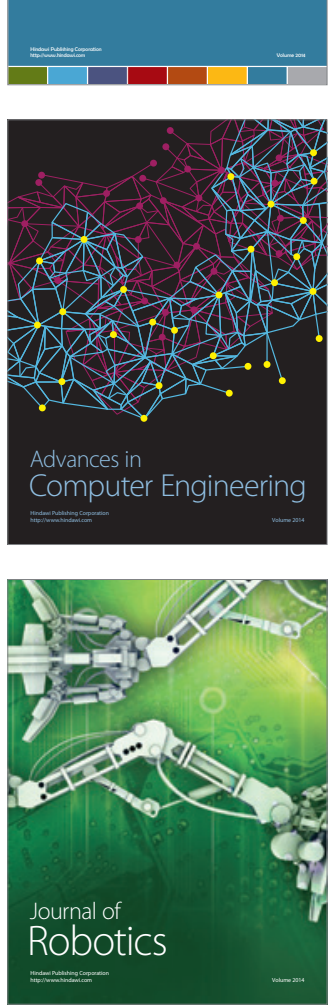
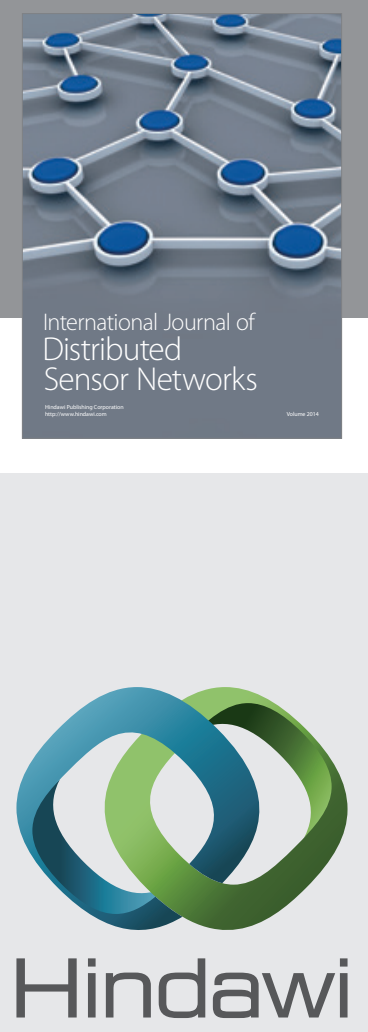

Submit your manuscripts at

http://www.hindawi.com
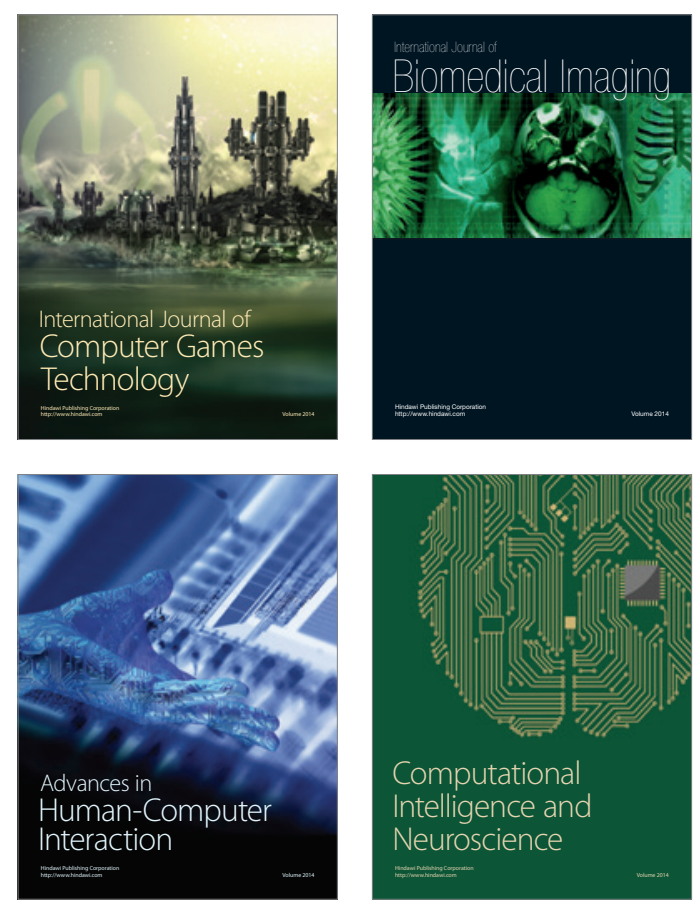
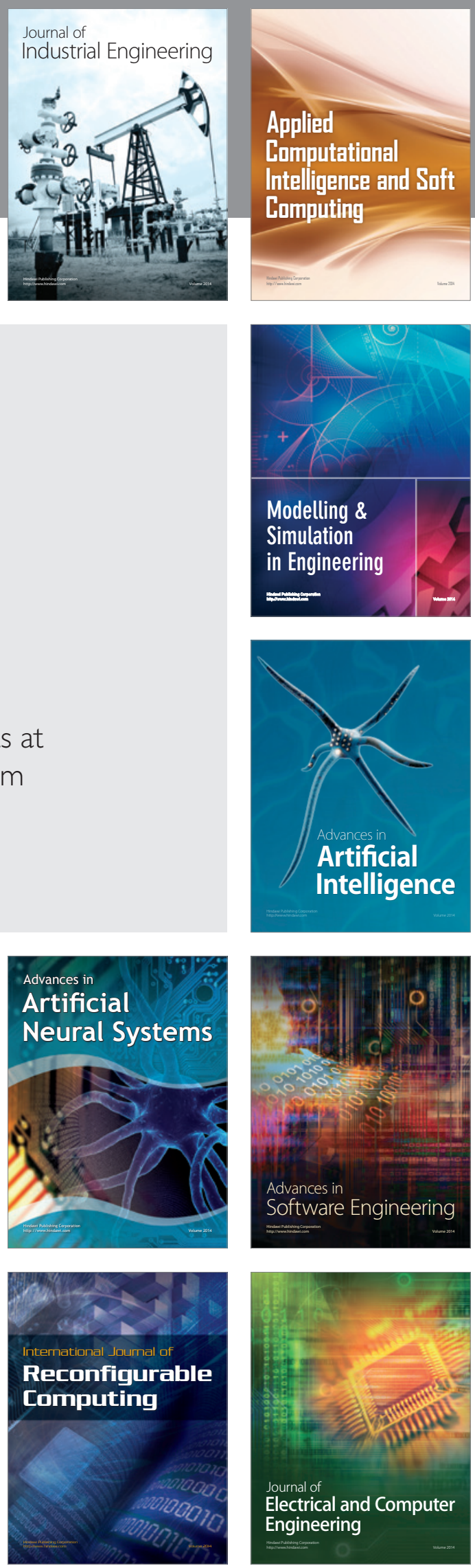